\title{
Targeting the extrinsic apoptotic pathway in cancer: lessons learned and future directions
}

\author{
Avi Ashkenazi \\ Cancer Immunology, Genentech Inc., South San Francisco, California, USA
}

\begin{abstract}
Apoptosis is a metazoan process of controlled cell elimination that plays critical roles in embryonic development and adult tissue homeostasis. Apoptosis dysregulation contributes to several important diseases, including cancer. Two distinct yet interconnected signaling pathways control apoptosis by activating a core intracellular machinery of death proteases called caspases. The intrinsic apoptotic pathway engages caspases via members of the BCL-2 protein family and the mitochondria in reaction to severe cellular damage or stress. The extrinsic pathway activates caspases via cell-surface death receptors, which respond to cognate death ligands expressed on immune-effector cells. Tumor cells can acquire various apoptosis-evasion mechanisms; nevertheless, the transformed state of these cells makes them uniquely susceptible to apoptosis reactivation if resistance is circumvented. Molecular approaches to reengage the apoptotic pathways in cancer have been underway for over two decades. Gratifyingly, BCL-2 antagonists - which drive the intrinsic pathway - are beginning to bear clinical fruit. In contrast, clinical attempts to stimulate the extrinsic pathway with proapoptotic receptor agonists (PARAs) have been disappointing, despite compelling preclinical efficacy with this class of agents. Here, I discuss some of the possible reasons for this translational discrepancy and suggest strategies to overcome it with the next generation of PARAs.
\end{abstract}

\section{From Coley's toxins to PARAs}

In 1894, Coley showed that cell extracts from gram-negative bacteria caused tumor shrinkage in patients. Nearly a century later, the discovery and cloning of TNF- $\alpha$ as a host factor induced by bacterial LPS made it possible for the first time to attempt to recapitulate Coley's seminal observation with a single, molecularly defined agent. Although purified TNF- $\alpha$ was too toxic for systemic therapy, it was later approved in Europe for the treatment of sarcoma by isolated limb perfusion (1). Subsequently, Krammer and Nagata identified the death receptor Apo1/Fas (CD95), which helped decipher the extrinsic apoptotic pathway (2-5). However, attempts to activate CD95 for cancer therapy were again hampered by toxicity, in this case due to excessive apoptosis of hepatocytes $(2,3)$.

A third opportunity to test the concept that tumor cells could be killed deliberately through a specific biological pathway arose in the mid-1990s, when my laboratory at Genentech and Ray Goodwin's laboratory at Immunex independently discovered another death ligand, called Apo2 ligand or TNF-related apoptosis-inducing ligand (Apo2L/TRAIL or TNFSF10) $(6,7)$. My team developed a recombinant soluble version of the human ligand comprising the extracellular domain of the endogenous protein and possessing a homotrimeric structure. In our 1999 JCI article (8), we reported that recombinant Apo2L/ TRAIL induced apoptosis in a wide range of cancer cell lines while sparing various

Conflict of interest: The author is an employee of Genentech Inc., a member of the Roche Group, and an owner of Roche restricted stock.

Reference information: / Clin Invest. 2015;125(2):487-489. doi:10.1172/JCI80420.

normal cell types. Moreover, the recombinant ligand exerted significant antitumor activity as a single agent and in combination with chemotherapy in a murine cancer xenograft model (8). The Immunex group reported similar results with a version of the ligand that was trimerized via a yeast-Gal4 leucine zipper (9). These findings were corroborated and expanded in numerous studies (10-12). X-ray crystallography later revealed that stabilization of the homotrimeric Apo2L/TRAIL molecule by an internal zinc ion was crucial for its selective proapoptotic activity against malignant, but not normal, cells $(13,14)$. The work with Apo2L/TRAIL and the identification of its cognate proapoptotic death receptors DR4 (TNFRSF10A) and DR5 (TNFRSF10B) $(4,15)$ prompted several groups, including my own, to develop agonistic anti-DR4 and anti-DR5 antibodies $(16,17)$. Compared with soluble Apo2L/ TRAIL, these antibodies enable less frequent dosing; however, the agonistic activity of anti-DR4 and anti-DR5 antibodies in vivo is restricted by a requirement for binding to $\mathrm{Fc} \gamma$ receptors (18). Conversely, cross-linking of Apo2L/TRAIL - either directly or via anti-DR5 antibody - triggers apoptosis in tumor-associated endothelial cells, disrupting the tumor vasculature while sparing normal vessels $(19,20)$. Thus, optimizing geometry and stoichiometry of PARAs appears crucial for effective and selective apoptosis engagement.

The impressive efficacy of PARAs in preclinical cancer models provided a compelling rationale for testing these agents in the clinic. To date, about 30 phase I and/ or phase II trials have been conducted to evaluate PARAs in various cancers, including non-small-cell lung cancer, colorectal cancer, pancreatic cancer, multiple myeloma, and non-Hodgkin's lymphoma (http://www.clinicaltrials.gov). Importantly, unlike TNF- $\alpha$ and anti-CD95 agonist antibodies, Apo2L/TRAIL and DR4or DR5-targeting agonist antibodies were relatively well tolerated. Disappointingly, 


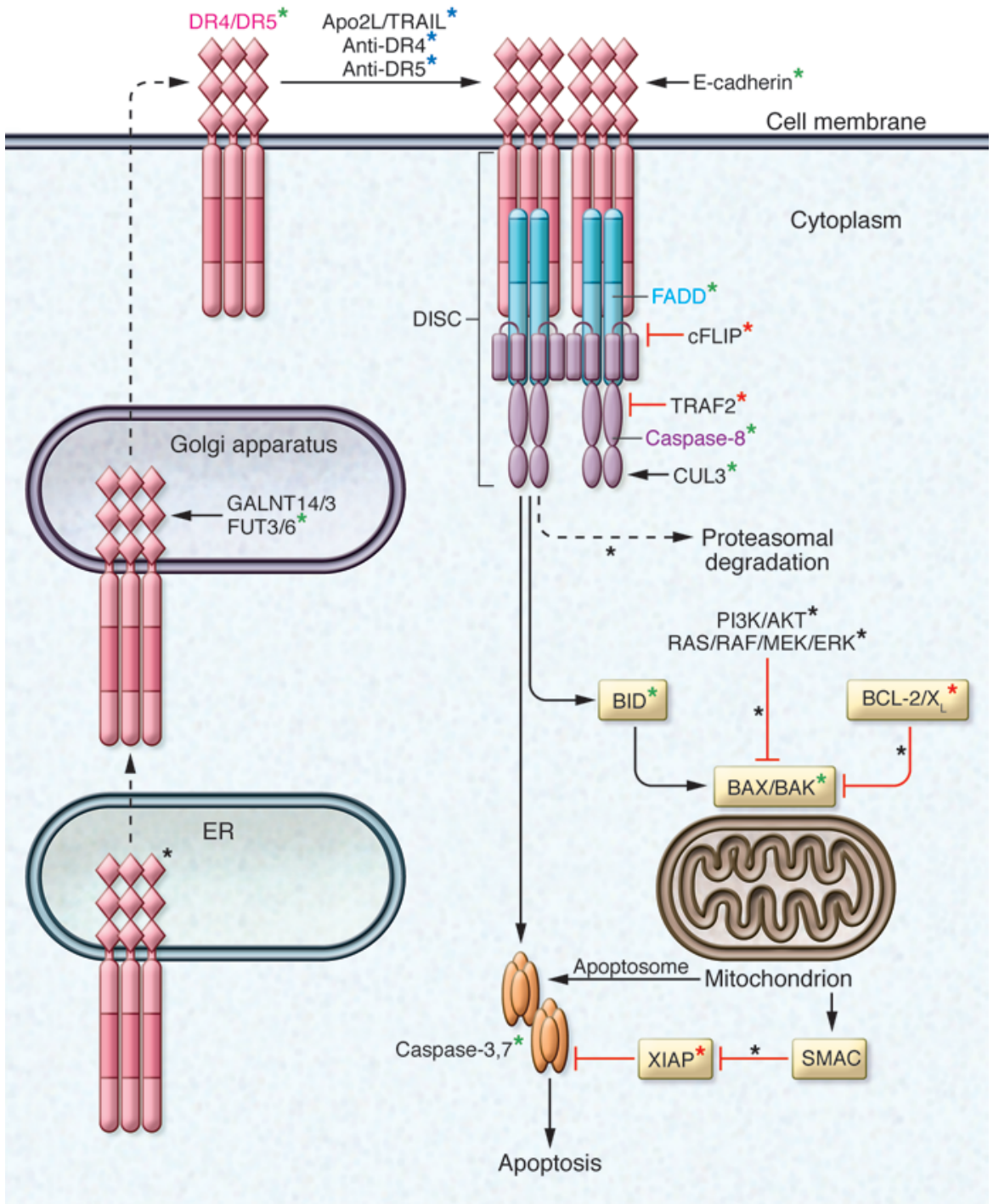

these PARAs failed to show significant efficacy either as monotherapies or in combination with conventional chemotherapies and/or certain biological agents. There were some rare, yet notably durable positive responses, for example, in a patient with chondrosarcoma (21).

\section{Lessons learned and future strategies}

What might account for the discrepant preclinical and clinical results with PARAs? One plausible explanation is that tumors encountered in the clinic have a higher threshold for reactivation of the extrinsic pathway than do those in preclinical models. This potentially could be addressed by implementing several strategies (Figure 1): (a) augmenting PARA potency a step beyond the first generation of agents while ensuring a favorable therapeutic index; (b) stratifying patients and optimizing dosing based on predictive and pharmacodynamic ing PARAs with other targeted agents to achieve synthetic lethality against tumors. Approaches to augment potency include the presentation of two Apo2L/ TRAIL trimers on $\mathrm{Fc}$ fusion platforms (22) or multiple trimers on liposomal membranes (P. Nair and A. Ashkenazi, unpublished observations), or a combined treatment with Apo2L/TRAIL and a suitable DR5 antibody (20). Biomarkers that may help predict responsiveness to PARAs include membranous expression of DR4 and DR5 on malignant and endothelial cells within tumors; O-glycosylation enzymes involved in post-translational modification of DR4 and DR5 in the Golgi apparatus - a modification diagnostic biomarkers; and (c) combin-

Figure 1. Apoptotic signaling pathways engaged by PARAs that target the death receptors DR4 and DR5. There are several potential strategies to improve the clinical efficacy of these PARAs. One strategy would be to augment potency by increasing the oligomeric state of Apo2L/TRAIL or the affinity of agonistic anti-DR4 or anti-DR5 antibodies for Fc $\gamma$ receptors (blue asterisks). Another strategy to improve efficacy would be the implementation of predictive and pharmacodynamic diagnostic biomarkers that might help predict or determine whether a patient's cancer is sensitive (green asterisks) or resistant (red asterisks) to PARA treatment. A third approach would be to improve synthetic lethality against cancer cells by combining PARAs with pharmacological agents that target various other intracellular signaling components or modulators of the apoptotic pathways (black asterisks). BAX/BAK, BCL-2-associated $X$ protein/BCL-2 antagonist killer 1; BCL-2/ $\mathrm{X}_{\mathrm{L}}$, B cell lymphoma-2/extra long; $\mathrm{BID}, \mathrm{BH} 3$-interacting domain death agonist; cFLIP, cellular FLICE-inhibitory protein; CUL3, cullin 3; DISC, death-inducing signaling complex; FADD, Fas-associated death domain; FUT3/6, fucosyltransferase 3/6; GALNT14/3, polypeptide $\mathrm{N}$-acetylgalactosaminyltransferase $14 / 3$; SMAC, second mitochondria-derived activator of caspases; TRAF2, TNF receptor-associated factor 2; XIAP, X-linked inhibitor of apoptosis.

that augments ligand-induced receptor clustering $(23,24)$; Fc $\gamma$ receptor polymorphism (18), which may impact the affinity and hence efficacy of agonistic antibodies; expression of E-cadherin, which facilitates ligand activation of DR4 and DR5 by dynamically coupling these receptors to the actin cytoskeleton in epithelial cancer cells (25); and ubiquitin E3 ligases involved in potentiating or curtailing caspase-8 activation in epithelial cancer cells $(26,27)$, among other components and modulators of the extrinsic pathway. Pharmacodynamic biomarkers may include cleaved caspase- 8 and caspase- 3 or other readouts for caspase activation and apoptosis. Potential synthetic lethal strategies include combinations with BCL-2 antagonists, IAP antagonists, proteasome inhibitors, agents targeting aberrant signaling cascades such as the 
RAS/RAF/MEK/ERK, PI3K/AKT, JNK, or p38 MAPK pathways (28), or inducers of ER stress (29). Finally, it would also be interesting to explore whether PARAs cooperate with the emerging class of cancer immunotherapeutic agents.

\section{Conclusions}

An attractive feature of apoptosis reactivation is the potential to cause tumor regression rather than just stasis. On the other hand, discriminating between malignant and healthy cells is crucial to avoid untoward side effects. Attempts to translate Coley's seminal findings by directly engaging the extrinsic apoptotic pathway were hampered by toxicities associated with TNF- $\alpha$ or anti-CD95 antibodies. We now have progressed beyond these safety hurdles with DR4- and DR5-targeted PARAs, although clinical efficacy with these agents has yet to be achieved. This creates a unique opportunity to overcome tumor resistance by (a) developing second-generation PARAs with enhanced potency while maintaining a therapeutic index; (b) implementing diagnostic biomarker approaches; and (c) investigating more advanced combinatorial strategies. Albert Einstein said: "failure is success in progress." I hope that researchers in academia and industry will find this article helpful in their quest to harness the extrinsic pathway for medical benefit.

\section{Acknowledgments}

I am grateful to Scot Marsters, David Lawrence, Robert Pitti, Klara Totpal, Sharon Yee, Ralph Schwall, Susan Leung, Roger Pai, Heather Flores, Sean Kelly, Vishva Dixit, and Diane Marschang; to former and present postdoctoral research fellows in my laboratory; and to many other colleagues at Genentech and beyond for their important contributions to this endeavor.
Address correspondence to: Avi Ashkenazi, Cancer Immunology, Genentech Inc., 1 DNA Way, South San Francisco, California 94080, USA. Phone: 650.225.1853;

E-mail:aa@gene.com.

1. Eggermont AMM. TNF registered in Europe: Does TNF get a second chance? J Immunother. 2000;23(4):505-506.

2. Nagata S. Apoptosis by death factor. Cell. 1997;88(3):355-365.

3. Peter ME, Krammer PH. Mechanisms of CD95 (APO-1/Fas)-mediated apoptosis. Curr Opin Immunol. 1998;10(5):545-551.

4. Ashkenazi A, Dixit VM. Death receptors: Signaling and modulation. Science. 1998;281(5381):1305-1308.

5. Wallach D, et al. Tumor necrosis factor receptor and Fas signaling mechanisms. Annu Rev Immunol. 1999;17:331-367.

6. Pitti RM, et al. Induction of apoptosis by Apo-2 ligand, a new member of the tumor necrosis factor cytokine family. J Biol Chem. 1996;271(22):12687-12690.

7. Wiley SR, et al. Identification and characterization of a new member of the TNF family that induces apoptosis. Immunity. 1995;3(6):673-682.

8. Ashkenazi A, et al. Safety and antitumor activity of recombinant soluble Apo2 ligand. J Clin Invest. 1999;104(2):155-162.

9. Walczak H, et al. Tumoricidal activity of tumor necrosis factor-related apoptosis-inducing ligand in vivo. Nat Med.1999;5(2):157-163.

10. Ashkenazi A. Targeting death and decoy receptors of the tumour-necrosis factor superfamily. Nat Rev Cancer. 2002;2(6):420-430.

11. Ashkenazi A. Directing cancer cells to selfdestruct with proapoptotic receptor agonists Nat Rev Drug Discov. 2008;7(12):1001-1012.

12. Johnston RW, Frew AJ, Smyth MJ. The TRAIL apoptotic pathway in cancer onset, progression and therapy. Nat Rev Cancer. 2008;8(10):782-798.

13. Hymowitz SG, et al. Triggering cell death: The crystal structure of Apo2L/TRAIL in a complex with death receptor 5. Mol Cell.1999;4(4):563-571.

14. Lawrence D, et al. Differential hepatocyte toxicity of recombinant Apo2L/TRAIL versions. Nat Med. 2001;7(4):383-385.

15. Wilson NS, Dixit VM, Ashkenazi A, Death receptor signal transducers: Nodes of coordination in immune signaling networks. Nat Immunol. 2009;10(4):348-355.

16. Ashkenazi A, Herbst RS. To kill a tumor cell: The potential of proapoptotic receptor agonists. J Clin Invest. 2008;118(6):1979-1990.

17. Wiezorek J, Holland P, Graves J. Death receptor agonists as a targeted therapy for cancer. Clin Cancer Res. 2010;16(6):1701-1708.

18. Wilson NS, et al. An Fc $\gamma$ receptor-dependent mechanism drives antibody-mediated targetreceptor signaling in cancer cells. Cancer Cell. 2011;19(1):101-113.

19. Wilson NS, et al. Proapoptotic activation of death receptor 5 on tumor endothelial cells disrupts the vasculature and reduces tumor growth. Cancer Cell. 2012;22(1):80-90.

20. Graves JD, et al. Apo2L/TRAIL and the death receptor 5 agonist antibody AMG 655 cooperate to promote receptor clustering and antitumor activity. Cancer Cell. 2014;26(7): 1-13.

21. Subbiah V, et al. Targeting the apoptotic pathway in chondrosarcoma using recombinant human Apo2L/TRAIL (dulanermin), a dual proapoptotic receptor (DR4/DR5) agonist. Mol Cancer Ther. 2012;11(11):2541-2546.

22. Gieffers C, et al. APG350 induces superior clustering of TRAIL receptors and shows therapeutic antitumor efficacy independent of cross-linking via Fc $\gamma$ receptors. Mol Cancer Ther. 2013;12(12):2735-2747.

23. Wagner KW, et al. Death-receptor O-glycosylation controls tumor-cell sensitivity to the proapoptotic ligand Apo2L/TRAIL. Nat Med. 2007;13(9):1070-1077.

24. Soria JC, et al. Randomized Phase II study of dulanermin in combination with Paclitaxel, Carboplatin and bevacizumab in advanced non-small cell lung cancer. J Clin Oncol. 2011;29(33):4442-4451.

25. Lu M, et al. E-cadherin couples death receptors to the cytoskeleton to regulate apoptosis. $\mathrm{Mol}$ Cell. 2014;54(6):987-998.

26. Jin Z, et al. Cullin3-based polyubiquitination and p62-dependent aggregation of caspase-8 mediate extrinsic apoptosis signaling. Cell. 2009;137(4):721-735.

27. Gonzalvez F, et al. TRAF2 Sets a threshold for extrinsic apoptosis by tagging caspase- 8 with a ubiquitin shutoff timer. Mol Cell. 2012;48(6):888-899.

28. Yang A, Wilson NS, Ashkenazi A. Proapoptotic DR4 and DR5 signaling in cancer cells: toward clinical translation. Curr Opin Cell Biol. 2010;22(6):837-844.

29. Lu M, et al. Opposing unfolded-protein-response signals converge on death receptor 5 to control apoptosis. Science. 2014;345(6192):98-101. 